\title{
Variation of Azimuth Angle Distribution of EAS, With Slope of the Detector Array Plane - A Examination by Semi-Montecarlo Simulation
}

\section{K.Viswanathan}

\begin{abstract}
The azimuth angle distribution of EAS is expected, as Cosmic Rays are isotropic. It is seen that, if the plane of the detectors is not horizontal, the azimuth angle distribution becomes non-uniform. In such cases it is necessary to make proper correction for this non-uniformity, when one attempts to use the EAS data collected in such array, for source search. An attempt is made to correlate the extent of non- uniformity with the slope of the array plane, using simulation.
\end{abstract}

Key words: Cosmic Rays, Azimuth angle distribution, Zenith angle distribution, Extensive Air Shower.

\section{INTRODUCTION}

$\mathrm{O}_{\text {ne of the main aims of most of the cosmic ray }}$ experiments is to search for sources of UHE gamma rays. The minimum requirement for such an array is good angular resolution and pointing accuracy [1]. Angular accuracy of an array can be determined by analyzing the same showers using even numbered detectors and odd numbered detectors independently, and then comparing the two results $[2,3]$. Pointing accuracy is determined by looking for shadow of sun and moon in the cosmic rays [4,5]. Before proceeding for these, it is necessary to make sure that the estimation of the angle is correct. For this, the following distributions are checked. The zenith angle distribution is expected to peak around $20^{\circ}$. The projected angle distribution in the E-W and $\mathrm{N}-\mathrm{S}$ plane is expected to have a uniform distribution peaking at zero.

It is well known that the azimuth angle distribution of extensive air shower shows a uniform distribution. This is due to the fact that cosmic rays are isotropic. This is true in the case of an array of detectors which is plane (where there is no systematic variation of z-coordinates of detectors). It is seen that if the plane of the detectors is considerably different from horizontal, the azimuth angle distribution is no longer uniform. This is clearly seen in the case of the GRAPES III experiment [6] of TIFR at Cosmic Ray Laboratory, Ooty (Fig.1a). The zenith angle distribution is shown in Fig.1b.
Revised Manuscript Received on July 22, 2019.

* Correspondence Author

K.Viswanathan, Department of Physics, Kalasalingam Academy of Research and Education, Krishnankoil, Tamilnadu - 626126, India.

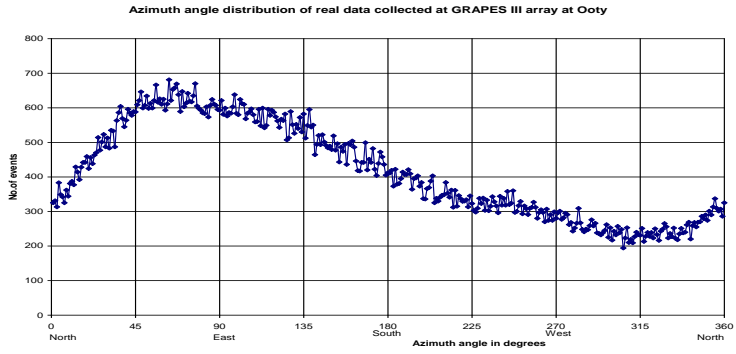

Fig. 1a Azimuth angle distribution of data collected at GRAPES III experiment at Ooty [6].

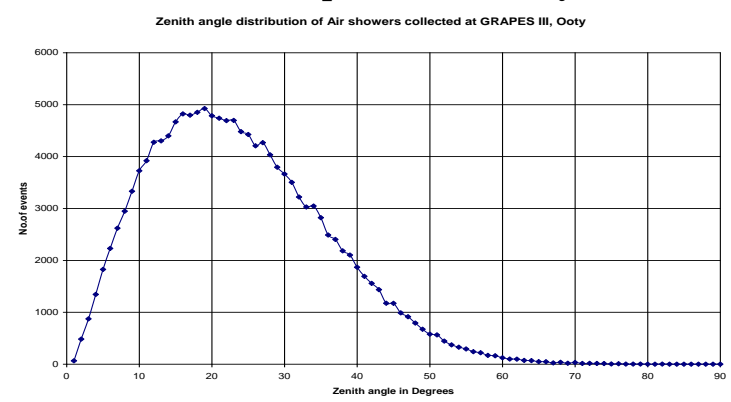

Fig. 1b Zenith angle distribution of CR showers collected at GRAPES III, Ooty [6]

In this paper, an attempt is made to study this variation systematically, using simulated data. I have generated EAS data using NKG function, for a hexagonal array of 217 detectors, similar to the GRAPES III array at Ooty, with varying slopes, and analysed the data. The azimuth angle distributions are plotted.

\section{SIMULATION}

An array of 217 detectors of each of area 1 square meter, was taken for the data simulation. The inter detector separation is taken as $8 \mathrm{~m}$. One million showers are generates using NKG function. The showers are generated with size ranging from $10^{4}$ to $10^{8}$, and age ranging from 0.8 to 1.2 . The variation of flux with zenith angle is assumed to be proportional to $\cos ^{7} \theta$, where $\theta$ is the zenith angle. The showers are generated with zenith angle varying from $0^{\circ}$ to $90^{\circ}$ and azimuth angle varying from $0^{\circ}$ to $360^{\circ}$.

Size and age are generated using random numbers, and the number of particles in each detector is estimated assuming a lateral distribution, which follows NKG (Nishimra-Kamata-Grieson) function:

$$
\Delta\left(N_{\alpha^{3}} s, r\right)=\frac{N_{0}}{2 \pi r} \cdot \frac{\Gamma(4.5-s)}{\Gamma(s) \cdot \Gamma(4.5-2 s)}\left(\frac{r}{r_{0}}\right)^{s-2}\left[1+\frac{r}{r_{0}}\right]^{(s-4.5)}
$$


where $\Delta$ is the density of particles in a detector which is at a distance $r$ from the core of the shower, $\mathrm{N}_{\mathrm{e}}$ is the size (total number of particles) of the shower, $r_{o}$ is the Molier unit of scattering length, $s$ is the age of the shower and $\Gamma$ is gamma function. Poisson and Gaussian fluctuations are also included for the densities.

The relative time of arrival of shower at different detectors are estimated using the relation

$$
\boldsymbol{l} \boldsymbol{x}_{i}+\boldsymbol{m} \boldsymbol{y}_{i}+\boldsymbol{n} z_{i}+\boldsymbol{c}\left(\boldsymbol{t}_{\bar{i}}-\boldsymbol{t}_{0}\right)=\mathbf{0}
$$

where $1, \mathrm{~m}$ and $\mathrm{n}$ are the direction cosines of the shower-axis, $\mathrm{x}_{\mathrm{i}}, \mathrm{y}_{\mathrm{i}}$ and $\mathrm{z}_{\mathrm{i}}$ are the coordinates of the $\mathrm{i}^{\mathrm{th}}$ detector, $\mathrm{c}$ is the velocity of the detector, $t_{i}$ is the relative time of arrival of the shower in $i^{\text {th }}$ detector and $t_{o}$ is the time of arrival of the shower at a fictitious detector at the core of the shower.

The first set (Set 0) of a million showers are generated for an array of detectors having same $\mathrm{z}$ coordinates (a flat array plane). Set 1 , Set 2 and Set 3 are generated for arrays sloping from east to west, at different angles. Set 4, Set 5 and Set 6 are for arrays sloping from North to south at three different angles. Set 7, Set 8 and Set 9 are for arrays sloping from South to North and Set 10, Set 11 and Set 12 for arrays sloping from west to east.

\section{DATA ANALYSIS}

The generated sets of data are analyzed for size, age and angle. The zenith angle distributions and azimuth angle distributions are plotted for the 13 sets separately. These are shown in Figs. 2 to 4 . It is clearly seen that as the slope increases, the variation from uniformity also increases. It can be noted that, there is a depletion in the number of showers from the direction where the $\mathrm{z}$-coordinates of the detectors are the highest. Similarly, there is an increase in the opposite direction. Even though it is not very well understood, the possible reason for this non-uniformity is the difference in the projected area of the array perpendicular to the arrival direction of the shower. The projected area of the detector plane in the direction of the shower is not the same or different azimuth angles of the same zenith angle. This causes a variation in the trigger efficiency of the detectors for showers of the same size coming from the zenith angle but different azimuth angles. If the array is perfectly horizontal, this variation will not be there. So, the effect is only experimental, and hence proper correction in the flux of showers has to be made before using the data for source search.

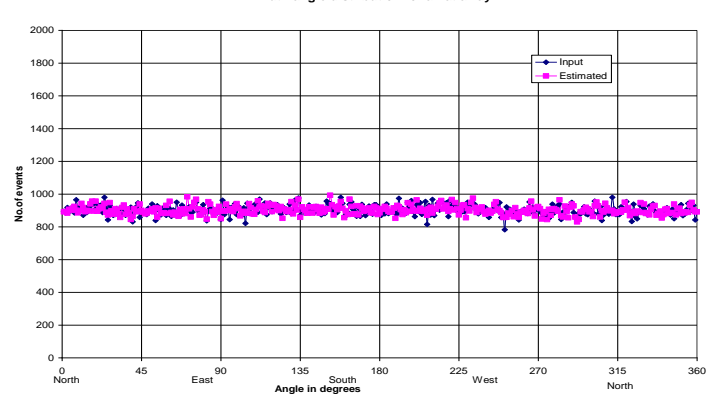

Fig.2 Azimuth angle distribution for a flat array

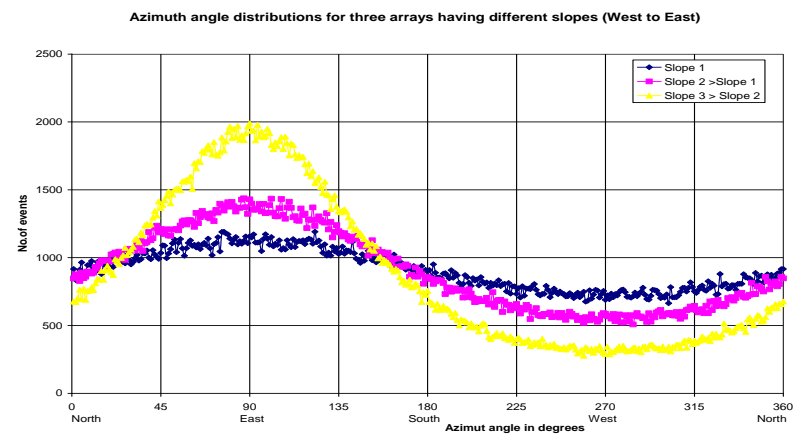

Fig.3 Azimuth angle distribution for arrays sloping from West to East

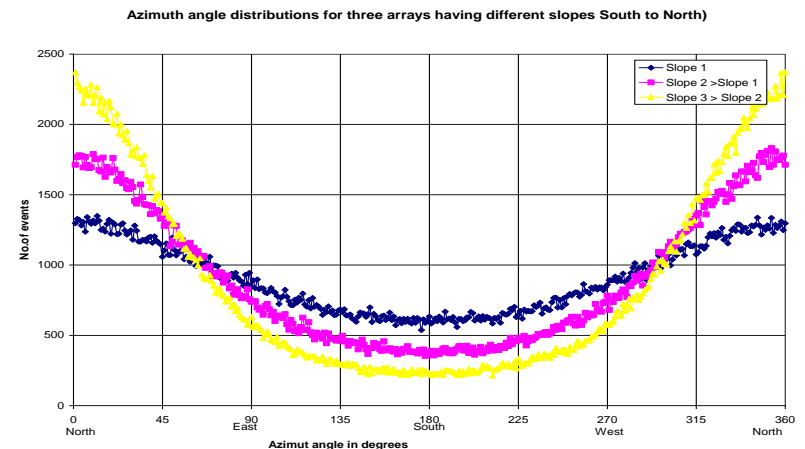

Fig.4 Azimuth angle distribution for arrays sloping from South to North

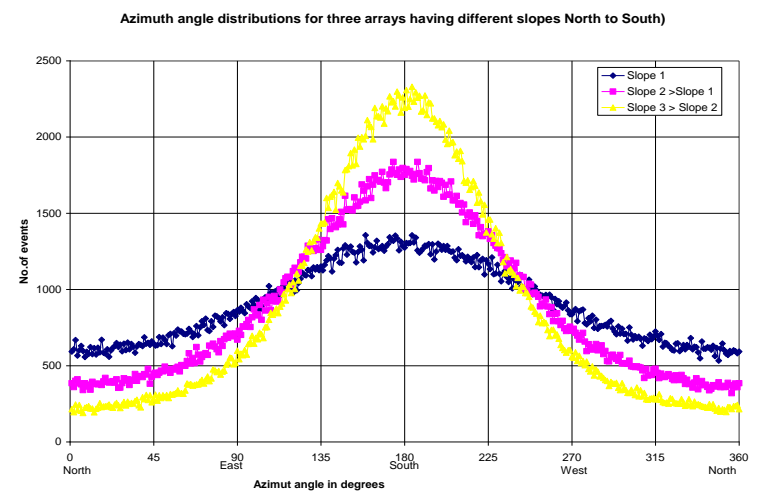

Fig. 5 Azimuth angle distributions for arrays sloping form North to South

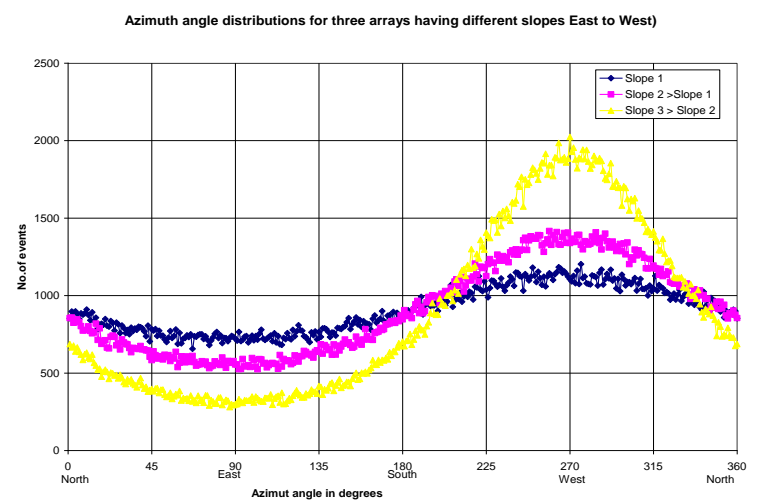

Fig. 6 Azimuth angle distributions for arrays sloping from East to West 


\section{CONCLUSIONS}

The variation of the azimuth angle distribution of the cosmic rays showers is found to have an effect on the slanting of the plane of the array of detectors. It can be inferred that the effect is due to the variation of the triggering efficiency of the shower with respect to the variation of projected area of the detector plane. More systematic study is in progress, and it is expected that a method to make correction for this non-uniformity can be obtained.

\section{ACKNOWLEDGMENT}

I sincerely thank all the members of Cosmic Ray group DHEP, TIFR, especially (Late) Prof.K.Sivaprasad, Prof.Suresh Tonwar, Prof. P.R.Viswanath, Prof.B.S.Acharya and Prof.Sunil Gupta, for their encouragement in this work.

\section{REFERENCES}

1. Apte A.R., Gopalakrishnan N.V., Tonwar S.C., Uma V., "Angular Resolution of an EAS Array for Gamma Ray Astronomy at $>5 \times 10^{13}$ eV", Proceedings of $19^{\text {th }}$ International Cosmic Ray Conference, Vol.3, 469-472, 1985

2. B.S.Acharya, P.N.Bhat, A.V.John, S.G.Khairatkar, B.K.Nagesh, M.R.Rajeev, K.Shobha Rao, M.V.S.Rao, V.A.Reddy, S.Sinha, K.Sivaprasad, A.J.Stanislaus, S.C.Tonwar, P.Unnikrishnan, S.S.Upadhyaya, B.L.Venkatesh Murthy, P.R.Viswanath, K.Viswanathan, "Angular Resolution of the K.G.F. experiment to detect Ultra High Energy Gamma Ray Sources”, Journal of Physics G., Nuclear and Particle Physics 19, 10531993.

3. Angular resolution of the GRAPES III EAS array for UHE Gamma ray Astronomy, P.K.Mohanty, S.K.Gupta, Y.Aikawa, N.V.Gopalakrishnan, Y.Hayashi, N.Ito, A.Iyer, A.Jain, P.Jagadeeshan, A.V.John, S.Karthikeyan, S.Kawakami, T.Matsuyama, D.K.Mohanty, S.D.Morris, T.Nonaka, A.Oshima, B.S.Rao, K.C.Ravindran, K.Sivaprasad, B.V.Sreekantan, H.Tanaka, S.C.Tonwar, K.Viswanathan, T.Yoshikoshi, Proceedings of $29^{\text {th }}$ International Cosmic Ray Conference, Pune, 6, 21 (2005).

4. K.Viswanathan, "Observation of Sun-Moon Shadow in Cosmic Rays, and determination of Angular resolution of the GRAPES III EAS Array", National Space Science Symposium, Kottayam, Kerala, 2004.

5. A. Oshima, S.R. Dugad, U.D. Goswami, S.K. Gupta, Y. Hayashi, N. Ito, A. Iyer, P. Jagadeesan, A. Jain, S. Kawakami, M. Minamino, P.K. Mohanty, S.D. Morris, P.K. Nayak, T. Nonaka, S. Ogio, B.S. Rao, K.C. Ravindran, H. Tanaka, S.C. Tonwar, "The angular resolution of the GRAPES-3 array from the shadows of the Moon and the Sun", Astroparticle Physics, 33, 97-107, 2010.

6. S.K. Gupta,Y. Aikawa, N.V. Gopalakrishnan, Y. Hayashi, N. Ikeda, N. Ito, A. Jain, A.V. John, S. Karthikeyan, S. Kawakami, T. Matsuyama, D.K. Mohanty, P.K. Mohanty, S.D. Morris, T. Nonaka, A. Oshima, B.S. Rao, K.C. Ravindran, M. Sasano, K. Sivaprasad, B.V. Sreekantan, H. Tanaka, S.C. Tonwar, K. Viswanathan, T. Yoshikoshi, "GRAPES-3 - A high-density air shower array for studies on the structure in the cosmic-ray energy spectrum near the knee", Nuclear Instruments and Methods in Physics Research A540, 311 (2005).

\section{AUTHORS PROFILE}

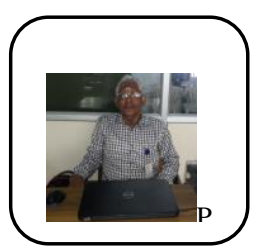

Dr.K.Viswanathan, Senior Professor, Department of Physics, Kalasalingam Academy of Research and Education, completed his M.Sc. and Ph.D. in Physics from University of Kerala, Trivandrum, and he has worked in the High Energy Cosmic Ray group of TIFR, at KGF and Ooty. He has around 60 publications in journals and same number of papers presented in International and National conferences. 